\title{
Small-scale chromospheric jets above a sunspot light bridge
}

\author{
Rohan E. Louis ${ }^{1}$, Christian Beck ${ }^{2}$, and Kiyoshi Ichimoto ${ }^{3}$ \\ ${ }^{1}$ Leibniz-Institut für Astrophysik Potsdam (AIP), An der Sternwarte 16, 14482 Potsdam, Germany \\ e-mail: rlouis@aip.de \\ 2 National Solar Observatory, Sacramento Peak, 3010 Coronal Loop, Sunspot, New Mexico 88349, USA \\ ${ }^{3}$ Kwasan and Hida Observatories, Kyoto University, Yamashina, 607-8471 Kyoto, Japan
}

Received 4 March 2014 / Accepted 30 May 2014

\begin{abstract}
Context. The chromosphere above sunspot umbrae and penumbrae shows several different types of fast dynamic events such as running penumbral waves, umbral flashes, and penumbral microjets.

Aims. The aim of this paper is to identify the physical driver responsible for the dynamic and small-scale chromospheric jets above a sunspot light bridge.

Methods. High-resolution broadband filtergrams of active region NOAA 11271 in Ca II H and G band were obtained with the Solar Optical Telescope on board Hinode. We identified the jets in the Ca II H images using a semi-automatic routine and determined their length and orientation. We applied local correlation tracking (LCT) to the $G$-band images to obtain the photospheric horizontal velocity field. The magnetic field topology was derived from a Milne-Eddington inversion of a simultaneous scan with the Spectropolarimeter. Results. The chromospheric jets consist of a bright, triangular-shaped blob that lies on the light bridge, while the apex of this blob extends into a spike-like structure that is bright against the dark umbral background. Most of the jets have apparent lengths of less than $1000 \mathrm{~km}$ and about $30 \%$ of the jets have lengths between $1000-1600 \mathrm{~km}$. The jets are oriented within $\pm 35^{\circ}$ to the normal of the spine of the light bridge. Most of them are clustered near the central part of the light bridge within a $2^{\prime \prime}$ area. The jets are seen to move rapidly along the light bridge and many of them cannot be identified in successive images taken with a 2 min cadence. The jets are primarily located on one side of the light bridge and are directed into the umbral core. The Stokes profiles at or close to the location of the blobs on the LB exhibit both a significant net circular polarization and multiple components, including opposite-polarity lobes. The magnetic field diverges from the light bridge towards the umbral cores that it separates. The LCT reveals that in the photosphere there is a predominantly uni-directional flow with speeds of $100-150 \mathrm{~m} \mathrm{~s}^{-1}$ along the light bridge. This unidirectional flow is interrupted by a patch of weak or very small motions on the light bridge which also moves along the light bridge.

Conclusions. The dynamic short-lived chromospheric jets above the LB seem to be guided by the magnetic field lines. Reconnection events are a likely trigger for such phenomenon since they occur at locations where the magnetic field changes orientation sharply and where we also observe isolated patches of opposite-polarity magnetic components. We find no clear relation between the jets and the photospheric flow pattern.
\end{abstract}

Key words. sunspots - Sun: chromosphere - Sun: photosphere - Sun: magnetic fields - techniques: photometric

\section{Introduction}

Light bridges (LBs) are bright structures that intrude and/or divide umbrae of sunspots and pores into multiple smaller umbral cores. Light bridges can have a penumbral or granular morphology (Muller 1979) and typically show a dark lane running along their central axis in high-resolution observations (Sobotka et al. 1994; Hirzberger et al. 2002; Berger \& Berdyugina 2003; Lites et al. 2004; Rimmele 2008). At the photosphere, LBs can be perceived as field-free intrusions of hot plasma into the gappy umbral magnetic field (Parker 1979; Choudhuri 1986) or largescale magneto-convective structures (Rimmele 2008). In either of the above cases, the LB forces the neighbouring umbral magnetic field to form a canopy around it which leads to a stressed magnetic configuration (Jurčák et al. 2006), particularly in the chromosphere and transition region. Such a topology has been suggested to be responsible for a wide variety of chromospheric activity such as surges in $\mathrm{H} \alpha$ (Asai et al. 2001), strong brightenings and ejections observed in Ca II H (Louis et al. 2008, 2009; Shimizu et al. 2009; Shimizu 2011), or brightness enhancements in the transition region (Berger \& Berdyugina 2003).

In this paper, we analyse the properties of small-scale chromospheric jets in a sunspot LB, using broadband filtergrams and spectropolarimetric data from Hinode (Kosugi et al. 2007). We relate the properties of the jets to the photospheric flow field and the magnetic field topology to identify what drives them and governs their evolution.

\section{Observations}

For this investigation, we utilised broadband filtergrams of the active region NOAA 11271 acquired by the Solar Optical Telescope (SOT, Tsuneta et al. 2008) on board Hinode. On 2011 August 19, the SOT observed the active region in the $G$ band (using a $0.8 \mathrm{~nm}$ filter centred at $430.5 \mathrm{~nm}$ ) and Ca II H (using a $0.3 \mathrm{~nm}$ filter centred at $396.9 \mathrm{~nm}$ ) with a spatial sampling of 0.11 and a cadence of $2 \mathrm{~min}$ for a duration of nearly $2.5 \mathrm{~h}$ from 8:05-10:33 UT. The field of view (FOV) of the filtergrams was $111^{\prime \prime} \times 111^{\prime \prime}$. The active region was located at a heliocentric angle $\Theta$ of $29^{\circ}$ at the time of the observations. The Ca II H images were recorded after the $G$-band images with a delay of $4 \mathrm{~s}$. All images were reduced with the standard routines of the Solar-Soft package. The images were subsequently co-aligned using a twodimensional cross-correlation routine.

At the same time, the FOV was scanned with the Hinode spectropolarimeter (SP; Ichimoto et al. 2008; Lites et al. 2013). 
During the time of the filtergram acquisition, the SP took five scans of the active region in the fast mode. Each scan covered a FOV of $75^{\prime \prime} \times 82^{\prime \prime}$. In the fast mode, the SP recorded the four Stokes profiles of the Fe I lines at $630 \mathrm{~nm}$ with a spectral sampling of $21.5 \mathrm{~m} \AA$, a step width of 0.29 , and a spatial sampling of 0.32 along the slit. Routines included in the Solar-Soft package were employed to reduce the Level 0 data (Lites \& Ichimoto 2013). We used the corresponding Level 2 maps of the vector magnetic field from MERLIN ${ }^{1}$ (Lites et al. 2007) inversions of the SP data. MERLIN uses a Milne-Eddington atmosphere where all physical parameters are constant with height except for the source function that varies linearly with optical depth. MERLIN assumes a single magnetic component and a variable stray light factor. The following parameters are retrieved by the code: field strength, inclination, azimuth, line-of-sight (LOS) velocity, damping constant, doppler width, line strength, macroturbulence, source function, source function gradient, and a stray light factor. The LOS observables were transformed to the localreference-frame. Prior to the transformation, the $180^{\circ}$ ambiguity in the azimuth was resolved manually by assuming a radial orientation of the transverse magnetic field and a spatially smooth azimuth.

\section{Data analysis}

\subsection{Local correlation tracking}

To determine if the proper motions have a role in the excitation of the chromospheric jets, we analysed the horizontal flow in the photosphere derived from local correlation tracking (November 1986; November \& Simon 1988; Welsch et al. 2004; Fisher \& Welsch 2008). Local correlation tracking (LCT) computes the relative displacement of small subregions centred on a particular pixel with subpixel accuracy using cross-correlation techniques. A Gaussian window, whose full width at half maximum needs to be roughly the size of the structures that are to be tracked, is used to apodize the subregions. Thus, the horizontal speed at each pixel can be determined knowing the displacement and the time interval. First, the $G$-band time sequence was filtered for acoustic waves using a phase velocity cutoff value of $7 \mathrm{~km} \mathrm{~s}^{-1}$ (Title et al. 1989). After several trials, an apodizing window with a width of $1^{\prime \prime}$ and a time difference of 2 min were chosen. Twenty successive velocity images were averaged to reduce the noise in the measurements. The LCT parameters are similar to the ones used in Vargas Domínguez et al. (2010) and Louis et al. (2012).

\subsection{Identification of chromospheric jets}

The top and bottom panels of Fig. 1 show the leading sunspot in active region (AR) NOAA 11271 in $\mathrm{Ca}$ II H and in the $G$ band, respectively. An animation of the $\mathrm{Ca} \mathrm{II} \mathrm{H}$ and $G$-band images of the full observation is available in the online section. The larger LB enclosed in the white rectangle was selected for the analysis. We chose the above active region because the LB was stable for a duration of more than $48 \mathrm{~h}$ and was located inside a regular sunspot. Because of the reduced $X$-band telemetry of Hinode after the end of 2007, the cadence of the imaging data was limited to allow for a reduction in data volume. Quantities such as lifetimes, proper motion and speeds could not be determined for most of the small-scale jets because they do not appear in

\footnotetext{
1 Level 2 maps from MERLIN inversions are provided by the Community Spectro-polarimetric Analysis Center at the link http:// www. csac. hao.ucar.edu/csac
}

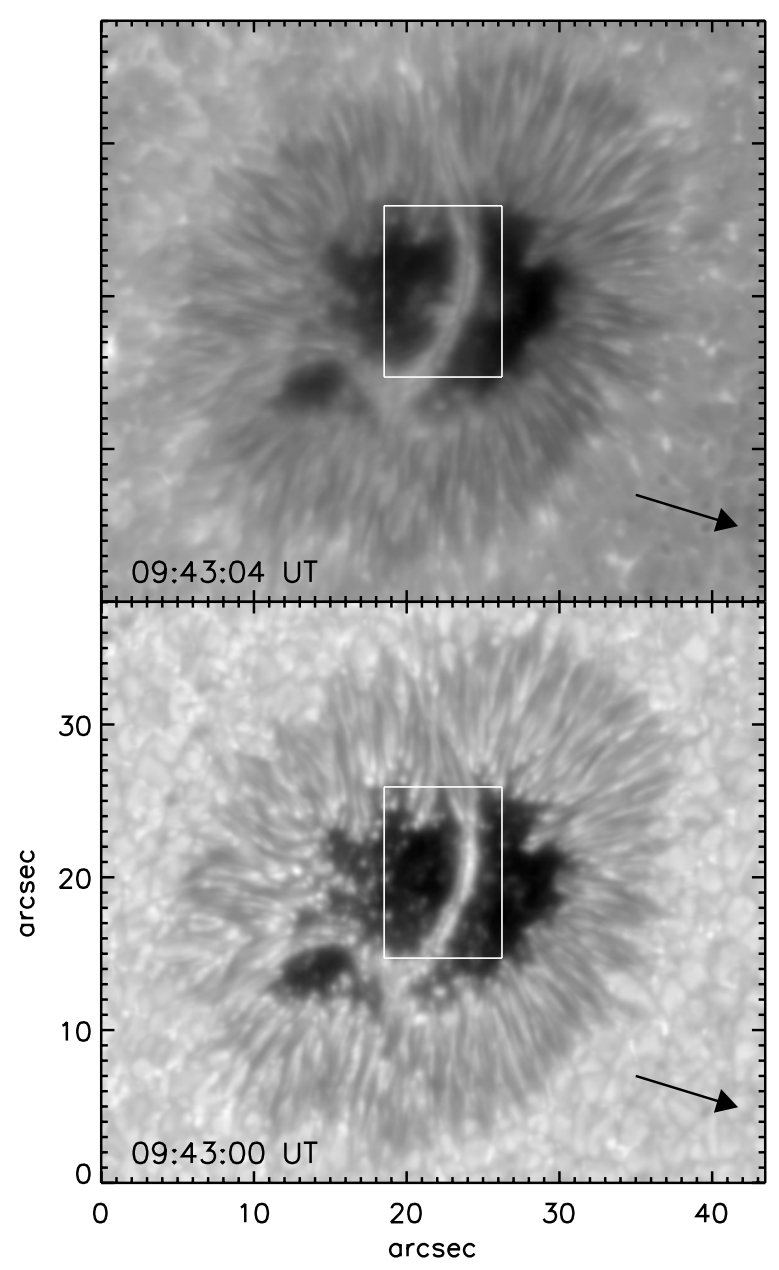

Fig. 1. Leading sunspot in NOAA AR 11271. The top and bottom panels correspond to the $\mathrm{Ca}$ II $\mathrm{H}$ and $G$ band, respectively. The large white rectangle represents the FOV selected for the analysis and shown in subsequent figures. The arrow points to disk centre. The images are displayed on a logarithmic scale.

consecutive frames (see Fig. 2). The animation of the time series shows a few longer-lived jets, as well as some cases where jets seem to move rapidly along the axis of the LB nearly in the same direction as the horizontal flow observed in the photosphere. Following the limitation caused by the temporal sampling, we focused on the characteristic properties of the jets, namely, position, length, and orientation as a statistical sample at an arbitrary time during their evolution.

The chromospheric jets on the LB are seen as a combination of bright, triangular blobs on the LB itself and spike-like structures which extend outward into the adjacent umbral background (Fig. 2). We refer to the blobs on the LB as the base of the jets. Identifying the jet required locating the base, followed by determining the orientation and length of the jet. The above steps were performed in a semi-automatic manner. Each Ca II H image was high-pass filtered using an 11-pixel boxcar as shown in the bottom panels of Fig. 2. The base of the jet located on the LB was selected by eye. A $7 \times 7$ pixel window centred on the selected position was used to determine the position of maximum intensity. Once the base of the jet was identified, the rest of the procedure was performed by the algorithm described below.

The orientation of the jet was determined by the angle at which the mean intensity along a specific radial distance from 
R. E. Louis et al.: Small-scale chromospheric jets above a sunspot light bridge
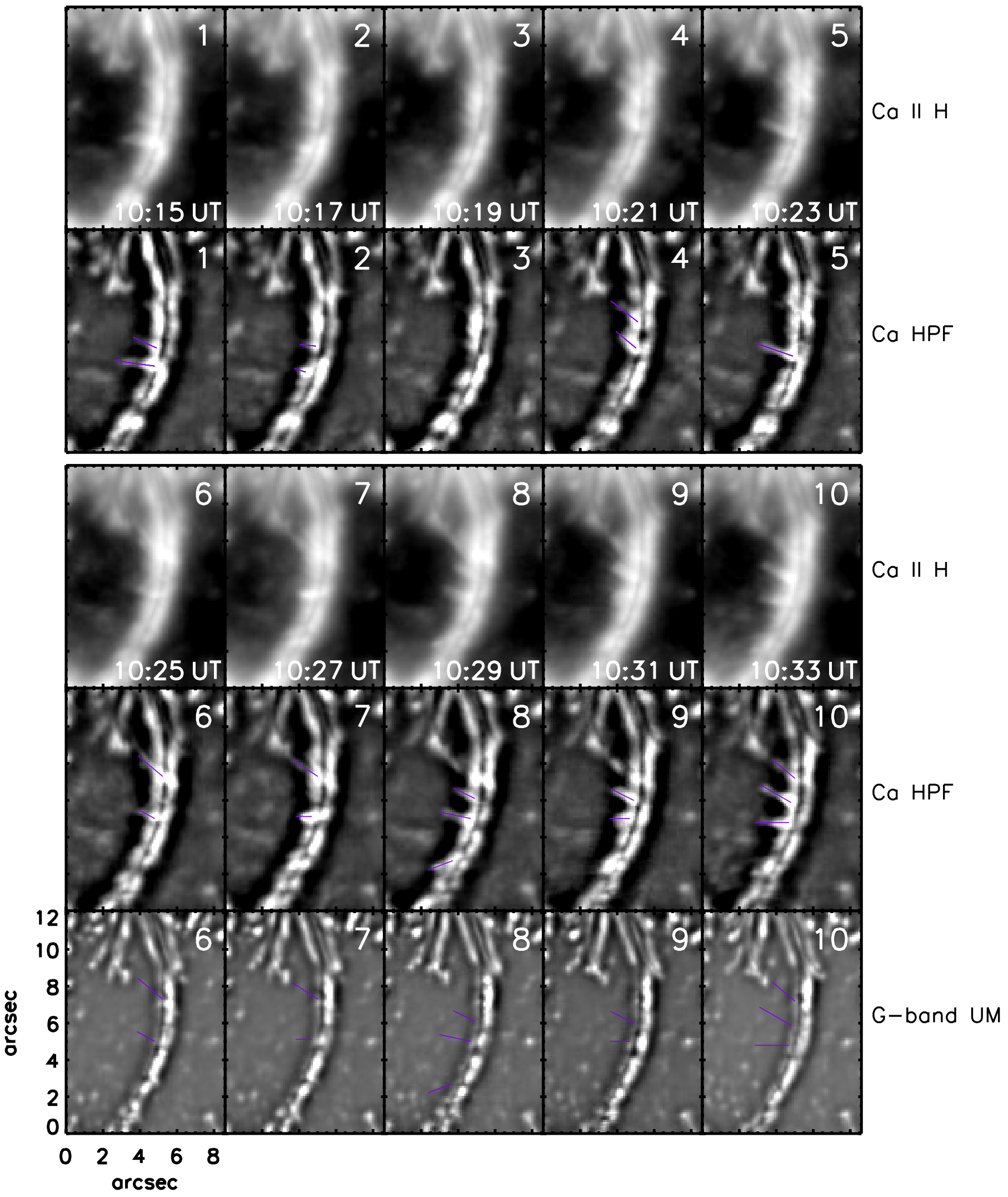

Fig. 2. Chromospheric jets seen in Ca II H. The top panels correspond to the $\mathrm{Ca}$ II $\mathrm{H}$ time sequence starting at 10:15 UT. The bottom panels are the corresponding high-pass filtered images (Ca HPF), with the purple lines representing the jets identified using the semi-automatic algorithm. The bottom panel shows unsharp masked $G$-band ( $G$-band $\mathrm{UM}$ ) images that correspond to the Ca II $\mathrm{H}$ time sequence shown in the panel above.

the base of the jet had a maximum value. To do this, we employed the loop-directivity procedure of Aschwanden (2010) that is part of a routine used for identifying coronal loops. The mean intensity along a given azimuthal direction $\left(\phi_{k}\right)$ for a fixed 
radial length $(L)$ is given by

$$
\begin{aligned}
I_{k} & =\frac{1}{L} \sum_{l=0}^{L} I\left(x_{k l}, y_{k l}\right), \text { where } \\
x_{k l} & =x_{b}+l \cos \phi_{k}, \text { and } \\
y_{k l} & =y_{b}+l \sin \phi_{k} .
\end{aligned}
$$

In the above, we assumed that the jet was a linear structure that extends from its base $\left(x_{b}, y_{b}\right)$ into the umbra. We set $L$ to 15 pixels and let $\phi_{k}$ vary from $140^{\circ}$ to $220^{\circ}$. The angle was measured relative to the $x$-axis and was limited to that range to avoid including the main body of LB itself because it would have created spurious results. The orientation $\left(\phi_{k}\right)$ of the jet is the angle $\phi_{k}$ for which $I_{k}$ is a maximum. Once the orientation was determined from the above procedure, the length of the jet was calculated from its base to the end of the jet where its intensity fell below a fixed threshold which was essentially the time-averaged umbral background derived from the high-pass filtered time sequence. In this manner, three parameters were determined for each jet in a given snapshot, i.e. the length, the orientation angle, and position. The jets identified in the sequence shown in Fig. 2 are depicted by purple lines that demonstrate the effectiveness of the above algorithm as well as the assumption that the extended part of the jets are linear. Since the primary selection of the jets was done by hand, there were several frames in the sequence that were skipped because the identification of jets was deemed uncertain (e.g. panel 3 in Fig. 2). We finally determined the orientation of the jet with respect to the normal of the LB axis. This axis was derived from the central dark lane that runs along the length of the LB that is clearly visible in the timeaveraged $\mathrm{Ca}$ II $\mathrm{H}$ image shown in Fig. 3. In the animation it can be seen that the location of the central dark lane was extremely stable the whole time with no change at all. We identified 87 jets in $75 \mathrm{Ca}$ II $\mathrm{H}$ images.

\section{Results}

\subsection{Properties of jets}

Although the basic characteristic of the chromospheric jets, described in the previous section and identified subsequently, is the spike-like structure with a bright blob on the LB, we could not identify or failed to detect any such feature in 20 snapshots spread over the observing run. Panel 3 in Fig. 2 is one such example where we find localised blobs of brightness on either side of the central dark lane, but these structures do not appear to have the essential property of a jet. We also find that such features without a spike exist on both sides of the central dark lane, but the jets we identify all lie on the eastern side, i.e. on the limb side of the LB. The Ca II H movie reveals that the blobs can also appear extended along the length of the LB. Some of them seem to move along the LB in the general direction of the photospheric velocity field (see Sect. 4.2 below), but there are also cases of motion in the opposite direction.

The top panel of Fig. 3 shows the positions of the bases of the jets on the LB with the colour indicating the frame number or time of the snapshot. With the exception of the two anchorage ends of the LB, the base of the jets are entirely distributed over its length, although there is a preferential clustering near the central part of the LB. The bottom panel of the figure indicates the apparent length and orientation of the jets and shows that these features extend from the LB to the eastern umbral core.

Figure 4 shows the histogram of the apparent length, orientation of the jets relative to the normal of the LB axis, and location

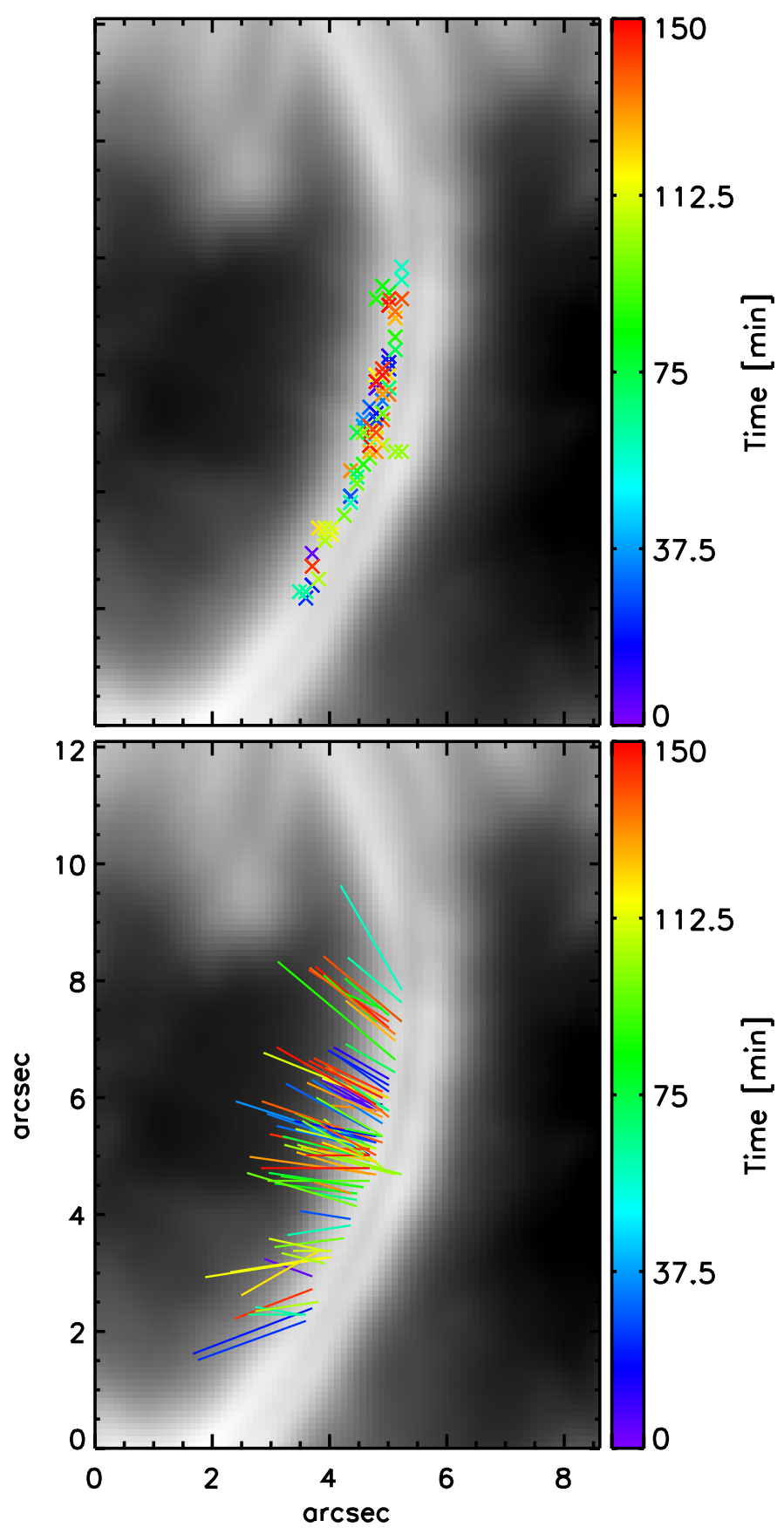

Fig. 3. Representation of the properties of the chromospheric jets detected over the observing period. The top panel shows the location of the base of the jet while the bottom panel indicates the length and orientation of the jet. The colours represent the time in the sequence when the jet was identified. The crosses and lines have been overlaid on the time-averaged $\mathrm{Ca}$ II $\mathrm{H}$ image.

with bin sizes of $150 \mathrm{~km}, 10^{\circ}$, and 0.5 , respectively. Most of the jets are shorter than $1000 \mathrm{~km}$ with the peak of the distribution lying around $750 \mathrm{~km}$. Nearly $30 \%$ of the jets detected have an apparent length between $1000-1600 \mathrm{~km}$. The relative orientation of the jets, indicated in the bottom panel shows that the jets lie within $\pm 35^{\circ}$ of the LB axis normal. Here, positive and negative values of the orientation in the figure refer to an orientation of the jet to the south and north of the normal to the LB axis, respectively. The location of the jets is measured along the central axis of the LB with $0^{\prime \prime}$ and $12^{\prime \prime} .9$ being at the bottom and 

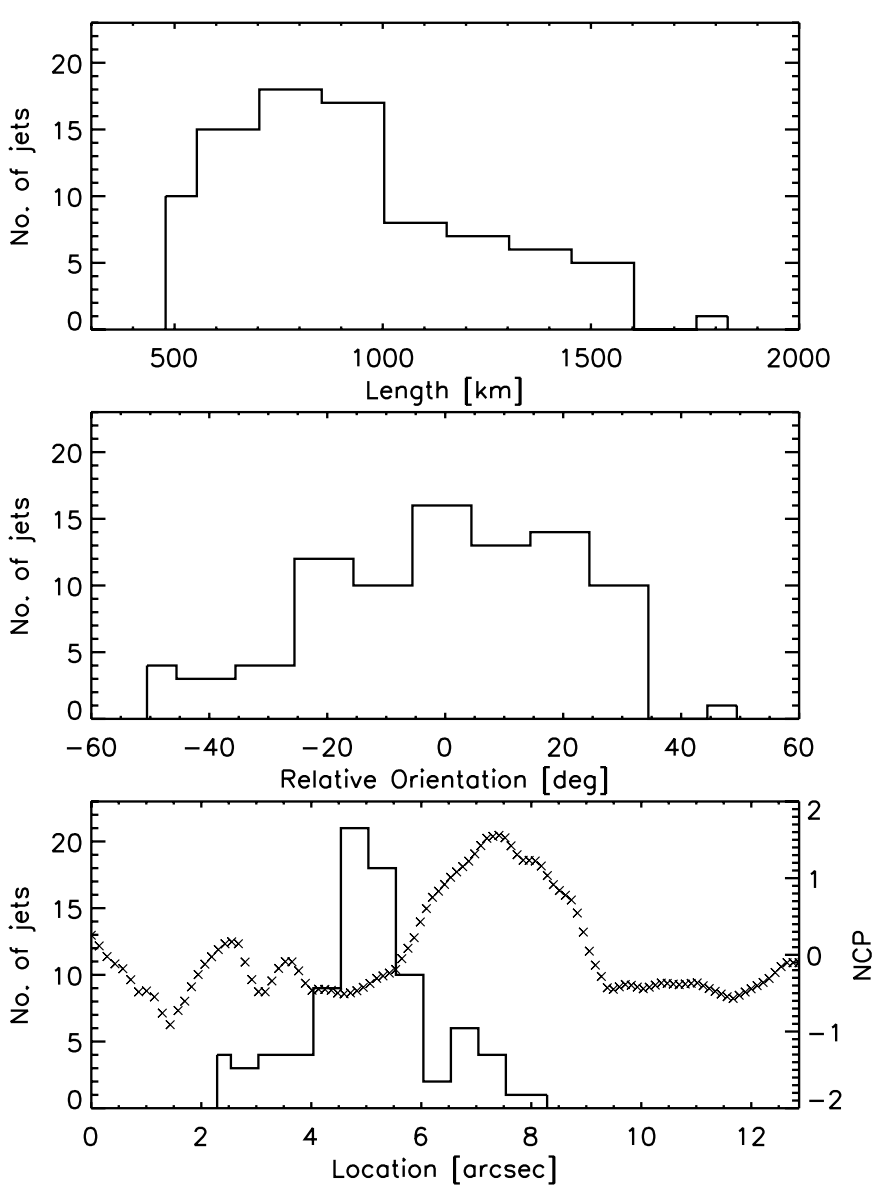

Fig. 4. Histogram of jet parameters. Top, middle, and bottom panels correspond to the length, relative orientation, and location of the jets, respectively. The bin sizes are $150 \mathrm{~km}, 10^{\circ}$, and $0{ }^{\prime} 5$, respectively. In the bottom panel the crosses refer to the net circular polarization along the centre of the LB with the scale given at the right-hand side of the plot.

top of the FOV, respectively. The bottom panel of Fig. 4 clearly shows a preferential clustering near the central part of the LB with nearly $65 \%$ of the jets located within a $2^{\prime \prime}$ area. The right $y$-axis refers to the net circular polarization along the centre of the LB (cf. Sect. 4.3 below).

\subsection{Horizontal flow in the photosphere}

The time sequence of $G$-band images shows that there is a patch of brightening that moves from the top to the bottom of the LB and lies predominantly on the right half of the LB. Small-scale structures in the $G$-band images also appear preferentially in this section of the light bridge. Faint striations are observed perpendicular to the central dark lane of the LB which get pushed along with the material flow. The dark lane in the $G$ band is fainter and is shifted more to the left edge of the LB while in the $\mathrm{Ca}$ II H images it is more prominent and centred on the LB. Although the chromospheric jets lack a one-to-one association with distinct features in the photosphere, they can sometimes be seen at or close to a bright grain on the LB ( $G$-band panels 7 and 9 in Fig. 2).

Figure 5 shows three horizontal flow maps obtained from LCT, each of which is an average of 20 maps covering a duration of $40 \mathrm{~min}$. Horizontal motions at the umbra-penumbra boundary have speeds of about $200-250 \mathrm{~m} \mathrm{~s}^{-1}$, while in the LB, they are about $100-150 \mathrm{~m} \mathrm{~s}^{-1}$. In all three maps, the flow is directed into the LB from its northern anchorage end to its southern end. However, the flow is neither uniform nor constant along the length of the LB. The flow pattern is interrupted by regions with very small or weak motions (see also the animation). The patch of weak flows is again followed by the downward flow towards the southern end. This patch of weak flows moves along the LB as it appears at the northern end and in the central part of the LB as seen in panels 1 and 2, respectively. The horizontal speeds at the southern end are much weaker than at the northern end where the southward flow along the LB encounters northward motions from the penumbra into the umbra which is similar to the findings of Louis et al. (2008). The predominantly north-to-south horizontal motion can be seen in the $G$-band movie which also reveals small-scale barb-like features in the LB that evolve on timescales of 10-20 min.

\subsection{Magnetic field topology of the $L B$}

Figure 6 shows the magnetic field strength, inclination, and LOS velocity derived from the inversion of the observed Stokes profiles. The figure also shows a map of the net circular polarization (NCP) determined by integrating Stokes $V$ over both spectral lines. The field strength in the LB varies along its length (top left panel). We find relatively weak magnetic fields of about $1-1.3 \mathrm{kG}$ extending into the $\mathrm{LB}$ at its upper and lower ends with some pixels showing field strengths of about $800 \mathrm{G}$ near its upper half. In the central part of the LB the field strengths are higher, ranging from $1.5-1.7 \mathrm{kG}$. We also find a very small region of weaker magnetic fields near the central part of the LB close to its left edge bordering the umbra. In the umbral cores, where the field orientation is nearly vertical, the field strength is about $2.5 \mathrm{kG}$. The inclination in the LB is about $140-150^{\circ}$ in the upper end of the LB, while in the central part of the LB the magnetic fields are relatively vertical. The location of the weak magnetic fields in the upper half of the LB coincides with more inclined magnetic fields, while in the central part the stronger fields are more vertical. The bottom right panel of Fig. 6 shows the LOS velocity with the LB exhibiting weak blueshifts but also redshifts of about $400 \mathrm{~m} \mathrm{~s}^{-1}$ at the location of the weak, inclined magnetic fields. At this location we also see that the NCP is nonzero within a large patch, which indicates the possible presence of vertical gradients in the magnetic field strength, inclination, and/or LOS velocity.

The left panel of Fig. 7 shows a Ca II H filtergram with the horizontal magnetic field overlaid on it. It is evident from the figure that the horizontal magnetic field diverges along the edges of the light bridge arising from the intrusion of hot plasma in the umbra. The diverging magnetic field at the edge of the LB is suggestive of a canopy (Jurčák et al. 2006). In addition, the magnetic field at the central part of the LB is oriented across the LB, while in the lower half of the LB the azimuth changes once again with the field directed along the LB. The disruption in the magnetic field also creates two azimuth centres in the two respective umbral cores adjacent to the light bridge. The right panel of Fig. 7 shows the photospheric continuum intensity map with the Stokes $V$ profile overlaid at every pixel of the image. While most of the profiles in the panel are normal profiles with antisymmetric lobes, there are also profiles with a weak third lobe in the blue wing (blue profiles) which are located near the upper half of the LB. This additional lobe has a polarity opposite that of the sunspot. We attribute the enhanced NCP (outlined by the purple contour) in this part of the LB to the presence of anomalous circular polarization signals. The bottom panel of Fig. 4 also indicates that the region of enhanced NCP lies immediately 

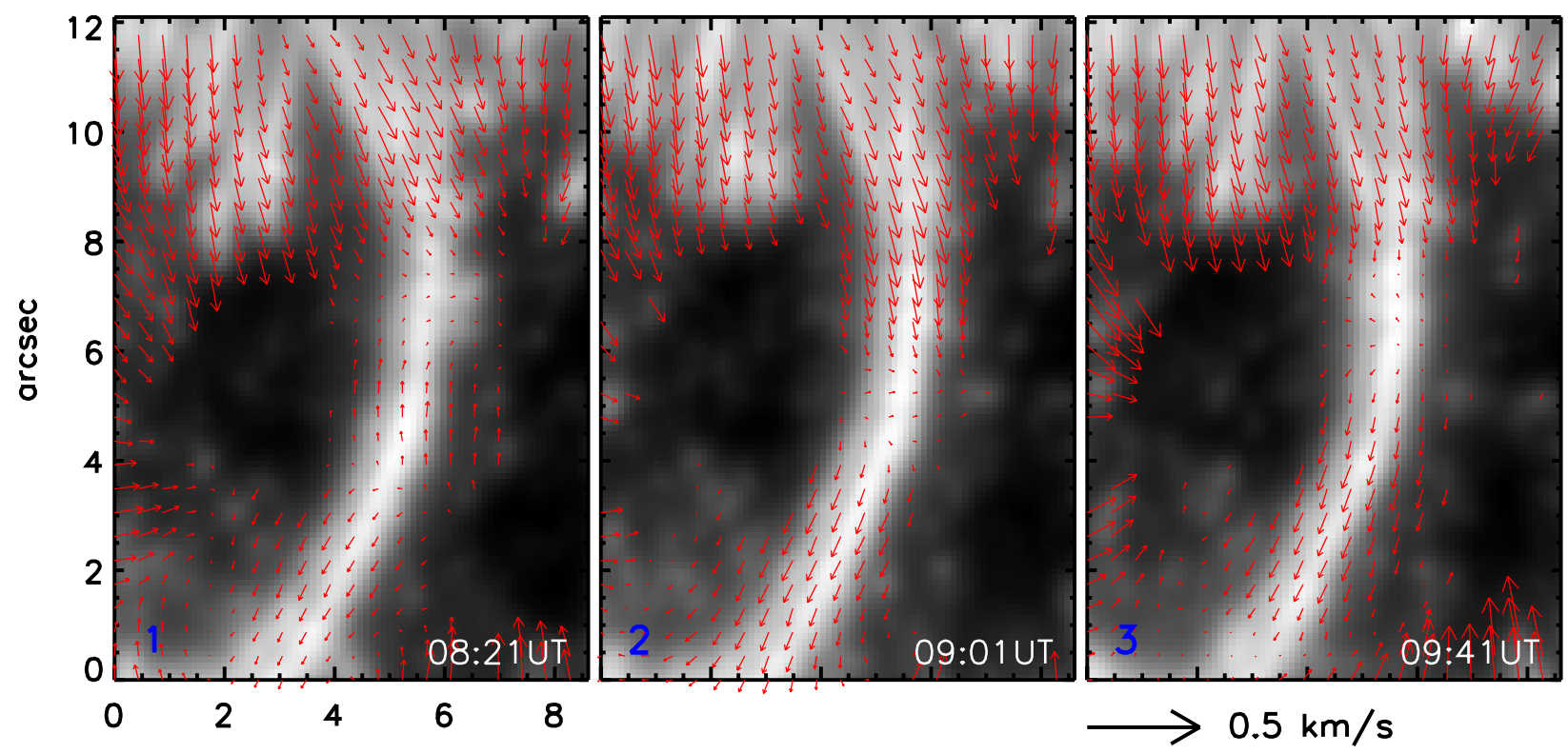

Fig. 5. Horizontal flow maps from LCT. The red arrows represent the horizontal flow field that was derived from averaging 20 flow maps spanning a duration of $40 \mathrm{~min}$. These have been overlaid on the corresponding time averaged $G$-band image.
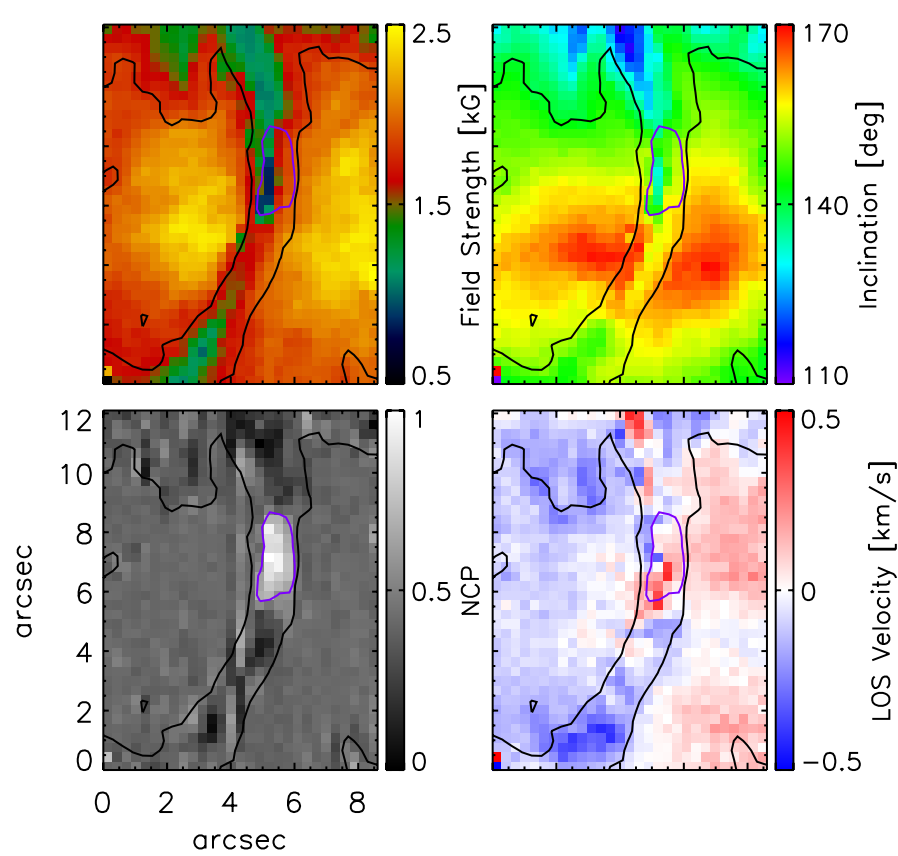

Fig. 6. Physical parameters in the light bridge. Clockwise from top left: magnetic field strength, field inclination, LOS velocity, net circular polarization. All maps have been scaled according to their colour bars. The maps correspond to the SP scan from 10:05-10:21 UT. The purple contour in the panels outlines a region of large NCP. The black contour outlines the light bridge and was derived from the continuum intensity image at $630 \mathrm{~nm}$ in the SP scan.

next to the location where a vast majority of the jets occur, while a few jets also originate from this patch. This suggests that the complex configuration of the magnetic field at this location is responsible for the preferential occurrence of the chromospheric jets. At the northern entrance of the LB, there are $V$ profiles that have a similar property, but here the third lobe appears on the red wing (red profiles). The blue and red coloured profiles suggest that the additional lobe is associated with a magnetic component that is strongly blue- and redshifted, respectively, by about 3-4 $\mathrm{km} \mathrm{s}^{-1}$. The strength of the lobe also implies that this additional component has a very small fill fraction. These pixels also extend out into the northern penumbra. Furthermore, we see $V$ profiles with reduced amplitudes (white profiles) that lie exclusively at the left edge of the LB and in the adjacent umbral core. The tiny patch of relatively weak fields near the left edge of the LB described above stems from these profiles. We also note that the maps shown in Fig. 6 were produced from a MilneEddington inversion with a single magnetic component. Such an inversion scheme cannot reproduce the spectral characteristics of the blue and red coloured profiles shown in Fig. 7 where one encounters gradients in the magnetic field and LOS velocity or multiple components in the resolution element (see Auer \& Heasley 1978; Sanchez Almeida \& Lites 1992; Beck 2011; Borrero \& Ichimoto 2011, and references in the last two articles).

\section{Discussion}

It is well known that the chromospheres of sunspots exhibit a number of dynamic phenomena. One of these is umbral flashes (UFs), sudden brightenings that are observed in the core of the chromospheric Ca II lines with a periodicity of about 3 min (Wittmann 1969). Umbral flashes are a result of waves propagating from the photosphere which turn into shocks on reaching the less dense chromosphere and are seen as blueshifted emission reversals in the core of the Ca II $8542 \AA$ line (Rouppe van der Voort et al. 2003; Centeno et al. 2006; Felipe et al. 2010). Although UFs are a large-scale phenomenon, it has been shown that within a resolution element there are at least two distinct magnetic components, one that has the same polarity as the spot with zero or slightly downflowing velocity, while the other is related to the shock wave that has a line core emission reversal and strong upflows (Socas-Navarro et al. 2000).

The other dynamic phenomenon in sunspots are ubiquitous, fine-scale jets called microjets (MJs) that are observed in the chromospheric penumbra (Katsukawa et al. 2007). Microjets have lifetimes of less than $1 \mathrm{~min}$ and widths of about $400 \mathrm{~km}$. Their lengths vary between $1000-4000 \mathrm{~km}$, although some MJs can have lengths of up to $10000 \mathrm{~km}$. It has been suggested that 

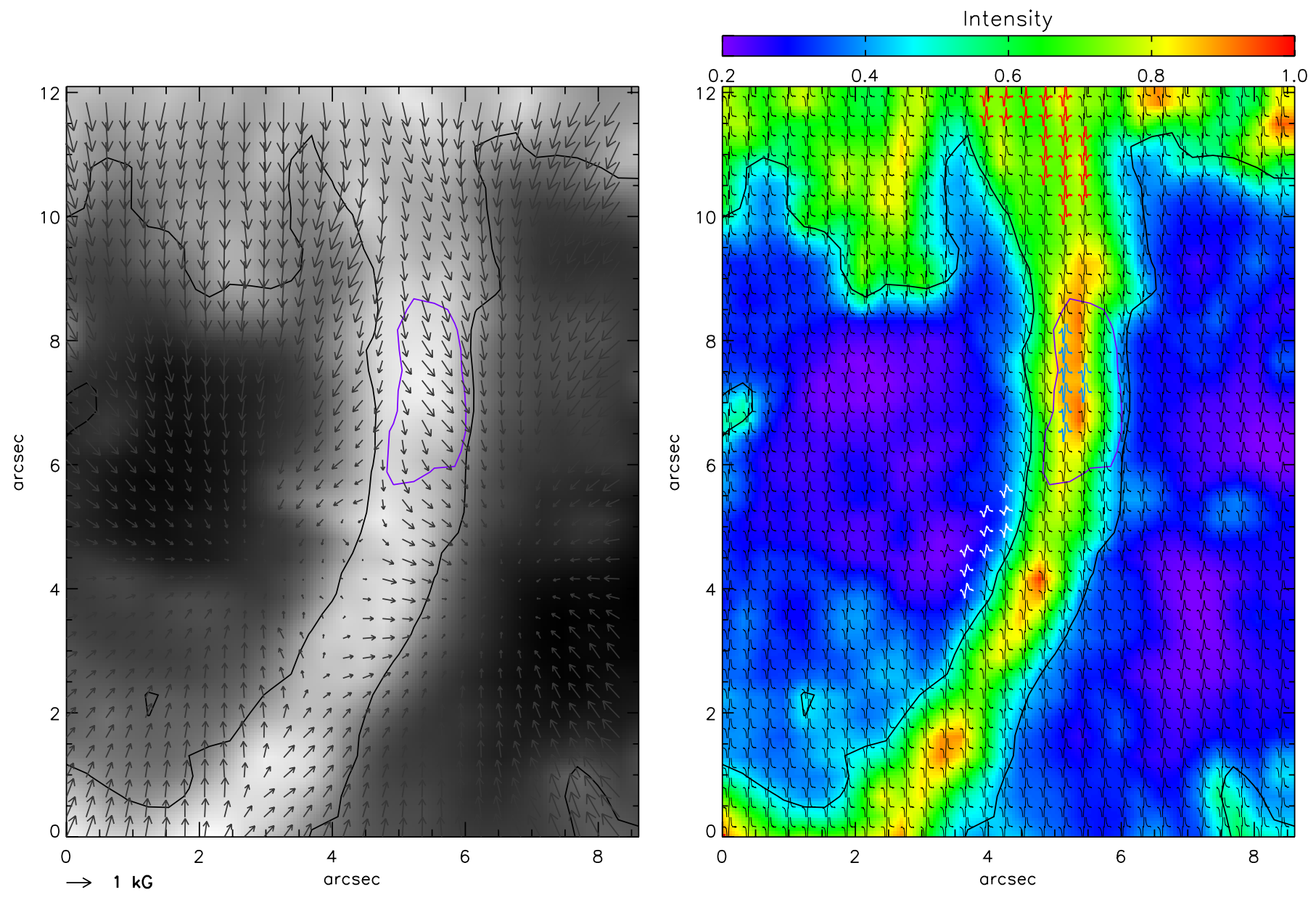

Fig. 7. Chromospheric jets and the photospheric magnetic field. The left panel shows the horizontal magnetic field from an SP scan with black arrows for every pixel and has been overlaid on a Ca II H filtergram taken 6 min after the SP slit was above the light bridge. The right panel shows the photospheric continuum intensity map in the background with the Stokes $V$ profiles overlaid on each pixel of the map. Only the $6302.5 \AA$ line has been shown and has been clipped to $\pm 8 \%$ of the quiet Sun continuum intensity. The purple contour outlines the region of strong NCP indicated in Fig. 6. See text for the description of the blue, red, and white profiles. The black contour outlines the light bridge and was derived from the continuum intensity image at $630 \mathrm{~nm}$ in the SP scan.

MJs are produced by reconnection in the intermediate region between a penumbral flux tube and the relatively vertical, background magnetic field as demonstrated in an MHD model by Magara (2010).

In a recent study of six penumbral transients Reardon et al. (2013) found that some of the events had spectral properties similar to those of Ellerman bombs (Ellerman 1917; Severny 1956; Fang et al. 2006), with enhanced emission in the wings of the Ca II $8542 \AA$ line. Other transients identified were possibly related to strong acoustic shocks, umbral flashes, or an emerging magnetic bipole in the chromosphere. Such emission features in the different parts of the spectral line show up as brightness enhancements in the broadband Hinode Ca II H filtergrams. From their observations, Reardon et al. (2013) suggest that the complexity and variety of the magnetic field structure in different regions of the sunspot is not only related to the distinct spectral characteristics of the transients, but also to the rapid timescales on which magnetic energy is released in the solar atmosphere.

The small-scale jets we have described could be produced by reconnection at the edge of the light bridge where the magnetic field is seen to diverge or wrap around it. The magnetic field also changes orientation along the length of the LB where the magnetic fields from the north encounter oppositely directed fields from the south near the central half of the light bridge, similar to the observations of Louis et al. (2009). Anomalous Stokes $V$ profiles having multiple components and lobes of opposite polarity are another indicator that the magnetic configuration in the LB is complex (see also Bharti et al. 2007). These profiles (coloured blue at the middle of the LB in Fig. 7) indicate the presence of strong gradients in the magnetic field and LOS velocity and/or multiple components in the resolution element. This location of enhanced net circular polarization lies in the immediate vicinity of where most of the jets occur, and strongly suggests that the stressed configuration of the magnetic field is responsible for the preferential occurrence of the chromospheric jets. Shimizu et al. (2009) showed that the presence of strong electric currents exclusively on one edge of a light bridge was responsible for chromospheric ejections being launched dominantly from that side of the light bridge. They interpreted the light bridge as a current-carrying twisted flux tube trapped below a cusp-shaped magnetic structure where strong currents were produced at the interface of the tube from fields having an antiparallel orientation with respect to the neighbouring umbral field.

In addition to the arrangement of the magnetic field, we also find a unidirectional, non-uniform flow that could facilitate the 
nearly persistent nature of the jets. Since LBs are a manifestation of weakly magnetised plasma disrupting the pre-existing nearly, homogeneous umbral magnetic field, the extent of the chromospheric activity could be related to the extent of the perturbation of the umbral magnetic field. This is demonstrated in individual case studies of LBs that at least show some related chromospheric activity at a certain stage of their evolution. On the other hand, the unidirectional, non-uniform flow in the light bridge could perturb the magnetic field in the light bridge exciting waves that propagate to the higher solar atmosphere, releasing the energy in the form of the jets. The coarse temporal sampling of the data and the limitations of our detection scheme mean that some of the jets are excluded from the study which could influence even static properties, namely the position, length, and relative orientation. Since we do not have the complete spectral line information, it is not possible to ascertain if the jets are associated with a line-core emission or an enhancement in the overall spectral intensity. We also note that a $0.3 \mathrm{~nm}$ filter is used for broadband Ca II H imaging, as is the case for Hinode, and primarily samples the higher photospheric layers less than $600 \mathrm{~km}$ in height, which is dominated by reverse granulation and not from higher chromospheric layers above $1000 \mathrm{~km}$, for observations at disc centre (Beck et al. 2013). Thus, even for our observations at a heliocentric angle of $30^{\circ}$, it is possible that the jets we have described are more upper photospheric in nature than fully chromospheric. Instruments such as the Blue Imaging Solar Spectrometer (BLISS, Puschmann et al. 2013) that has been planned at the $1.5 \mathrm{~m}$ GREGOR telescope (Schmidt et al. 2012), the Interferometric BIdimensional Spectrometer (IBIS; Cavallini 2006), and the CRisp Imaging SpectroPolarimeter (CRISP; Scharmer et al. 2008) will be crucial in understanding the mechanism responsible for these smallscale jets.

\section{Conclusions}

The small-scale, short-lived, chromospheric jets above the sunspot light bridge seem to be guided by the magnetic field. Reconnection events are a likely trigger for these phenomena since they occur at locations where the magnetic field changes orientation sharply. Localized patches of opposite-polarity magnetic components further illustrate that the magnetic field in the light bridge is complex which lends support to the reconnection scenario. While we do not find a clear relation between the chromospheric jets and the photospheric flow pattern, we do not rule out the possibility of the latter's role in perturbing the magnetic field and subsequently producing the jets through magnetic reconnection or by the excitation of waves. On the observational side, high-resolution spectroscopy in the chromosphere with high temporal resolution is crucial for determining the thermal and kinematic properties of such jets, which is not possible with our current broadband imaging observations.

Acknowledgements. Hinode is a Japanese mission developed and launched by ISAS/JAXA, collaborating with NAOJ as a domestic partner and NASA and STFC (UK) as international partners. Scientific operation of the Hinode mission is conducted by the Hinode science team organized at ISAS/JAXA. This team mainly consists of scientists from institutes in the partner countries. Support for the postlaunch operation is provided by JAXA and NAOJ (Japan), STFC (UK),
NASA, ESA, and NSC (Norway). Hinode SOT/SP Inversions were conducted at NCAR under the framework of the Community Spectro-polarimtetric Analysis Center (CSAC; http://www. csac.hao.ucar.edu/). R.E.L. is grateful for the financial assistance from the German Science Foundation (DFG) under grant DE $787 / 3-1$. We thank the anonymous referee for the useful comments.

\section{References}

Asai, A., Ishii, T. T., \& Kurokawa, H. 2001, ApJ, 555, L65

Aschwanden, M. J. 2010, Sol. Phys., 262, 399

Auer, L. H., \& Heasley, J. N. 1978, A\&A, 64, 67

Beck, C. 2011, A\&A, 525, A133

Beck, C., Rezaei, R., \& Puschmann, K. G. 2013, A\&A, 556, A127

Berger, T. E., \& Berdyugina, S. V. 2003, ApJ, 589, L117

Bharti, L., Rimmele, T., Jain, R., Jaaffrey, S. N. A., \& Smartt, R. N. 2007, MNRAS, 376, 1291

Borrero, J. M., \& Ichimoto, K. 2011, Liv. Rev. Sol. Phys., 8, 4

Cavallini, F. 2006, Sol. Phys., 236, 415

Centeno, R., Collados, M., \& Trujillo Bueno, J. 2006, ApJ, 640, 1153

Choudhuri, A. R. 1986, ApJ, 302, 809

Ellerman, F. 1917, ApJ, 46, 298

Fang, C., Tang, Y. H., Xu, Z., Ding, M. D., \& Chen, P. F. 2006, ApJ, 643, 1325

Felipe, T., Khomenko, E., Collados, M., \& Beck, C. 2010, ApJ, 722, 131

Fisher, G. H., \& Welsch, B. T. 2008, in Subsurface and Atmospheric Influences on Solar Activity, eds. R. Howe, R. W. Komm, K. S. Balasubramaniam, \& G. J. D. Petrie, ASP Conf. Ser., 383, 373

Hirzberger, J., Bonet, J. A., Sobotka, M., Vázquez, M., \& Hanslmeier, A. 2002, A\&A, 383, 275

Ichimoto, K., Lites, B., Elmore, D., et al. 2008, Sol. Phys., 249, 233

Jurčák, J., Martínez Pillet, V., \& Sobotka, M. 2006, A\&A, 453, 1079

Katsukawa, Y., Berger, T. E., Ichimoto, K., et al. 2007, Science, 318, 1594

Kosugi, T., Matsuzaki, K., Sakao, T., et al. 2007, Sol. Phys., 243, 3

Lites, B. W., \& Ichimoto, K. 2013, Sol. Phys., 283, 601

Lites, B. W., Scharmer, G. B., Berger, T. E., \& Title, A. M. 2004, Sol. Phys., 221,65

Lites, B., Casini, R., Garcia, J., \& Socas-Navarro, H. 2007, Mem. Soc. Astron. It., 78, 148

Lites, B. W., Akin, D. L., Card, G., et al. 2013, Sol. Phys., 283, 579

Louis, R. E., Bayanna, A. R., Mathew, S. K., \& Venkatakrishnan, P. 2008, Sol. Phys., 252, 43

Louis, R. E., Bellot Rubio, L. R., Mathew, S. K., \& Venkatakrishnan, P. 2009, ApJ, 704, L29

Louis, R. E., Ravindra, B., Mathew, S. K., et al. 2012, ApJ, 755, 16

Magara, T. 2010, ApJ, 715, L40

Muller, R. 1979, Sol. Phys., 61, 297

November, L. J. 1986, Appl. Opt., 25, 392

November, L. J., \& Simon, G. W. 1988, ApJ, 333, 427

Parker, E. N. 1979, ApJ, 234, 333

Puschmann, K. G., Denker, C., Balthasar, H., et al. 2013, Optical Engineering, 52,1606

Reardon, K., Tritschler, A., \& Katsukawa, Y. 2013, ApJ, 779, 143

Rimmele, T. 2008, ApJ, 672, 684

Rouppe van der Voort, L. H. M., Rutten, R. J., Sütterlin, P., Sloover, P. J., \& Krijger, J. M. 2003, A\&A, 403, 277

Sanchez Almeida, J., \& Lites, B. W. 1992, ApJ, 398, 359

Scharmer, G. B., Narayan, G., Hillberg, T., et al. 2008, ApJ, 689, L69

Schmidt, W., von der Lühe, O., Volkmer, R., et al. 2012, Astron. Nachr., 333, 796

Severny, A. B. 1956, The Observatory, 76, 241

Shimizu, T. 2011, ApJ, 738, 83

Shimizu, T., Katsukawa, Y., Kubo, M., et al. 2009, ApJ, 696, L66

Sobotka, M., Bonet, J. A., \& Vazquez, M. 1994, ApJ, 426, 404

Socas-Navarro, H., Trujillo Bueno, J., \& Ruiz Cobo, B. 2000, Science, 288, 1396

Title, A. M., Tarbell, T. D., Topka, K. P., et al. 1989, ApJ, 336, 475

Tsuneta, S., Ichimoto, K., Katsukawa, Y., et al. 2008, Sol. Phys., 249, 167

Vargas Domínguez, S., de Vicente, A., Bonet, J. A., \& Martínez Pillet, V. 2010, A\&A, 516, A91

Welsch, B. T., Fisher, G. H., Abbett, W. P., \& Regnier, S. 2004, ApJ, 610, 1148 Wittmann, A. 1969, Sol. Phys., 7, 366 